ARQGA / 1106

\title{
ALTERAÇÕES METABÓLICAS INDUZIDAS POR ISQUEMIA HEPÁTICA NORMOTÉRMICA EXPERIMENTAL E O EFEITO HEPATOPROTETOR DA CICLOSPORINA
}

José Huygens Parente GARCIA ${ }^{1}$, Gustavo Rego COELHO ${ }^{2}$, Ivian Teixeira de SOUSA ${ }^{2}$, Rafael Pontes de SIQUEIRA ${ }^{2}$ e Paulo Roberto Leitão de VASCONCELOS ${ }^{1}$

RESUMO - Racional - Transplante de fígado é inevitavelmente associado com períodos de isquemia completa. No entanto, o tempo de oclusão do pedículo hepático é limitado pelas consequiências da injúria pós-isquêmica do fíg ado. Objetivo - Deter minar as principais alterações metabólicas ocasionadas pela isquemia hepática e a provável ação hepatoprotetora da ciclosporina. Métodos - Isquemia hepática nor motérmica por 60 minutos foi induzida em ratos. Em seguida, as alterações com o tempo $(0,1,6,24$ horas $)$ das concentrações sangüíneas e hepáticas de lactato, piruvato, glicose, corpos cetônicos e razão acetoacetato/3-hidroxibutirato, bem como o estado redox citoplasmático e mitocondrial do tecido hepático foram determinados. Outro g rupo de animais foi pré-tratado com ciclosporina (10 mg/ $\mathrm{kg}$ ), sendo estudadas as alterações metabólicas no tempo 1 hora após revascularização hepática. Resultados - A isquemia hepática causou ele vação da concentração de lactato no fígado, sugerindo que pronunciado g rau de metabolismo anaeróbico ocorreu durante o período de isquemia. Isquemia hepática acar retou ainda queda da concentração e da razão dos corpos cetônicos (acetoacetato/3hidro xibutirato) no sangue arterial no tempo de 1 hora após revascularização. Tal fato reflete que a injuria isquêmica do fígado interfere na ceto gênese. Conclusão - O tratamento com ciclosporina causa elevação das concentrações dos corpos cetônicos e da razão acetoacetato/ 3-hidroxibutirato no sangue arterial após 1 hora de reperfusão hepática, sugerindo que esta droga acelera a cetogênese e, conseqüentemente, a recuperação da lesão isquêmica do fígado.

DESCRITORES - Fígado. Hepatectomia. Isquemia. Ciclosporina. Ratos.

\section{INTRODUÇÃO}

O transplante de fígado é atualmente uma realidade. Um dos problemas ainda presente é o período de isquemia completa, que pode acar retar lesões ir reversíveis neste órgão. O fígado humano pode suportar até 60 minutos de isquemia, sem aumento das complicações pós-operatórias, falência hepática ou mortalidade ${ }^{(4,7)}$. Apesar desses aspectos, o período máximo de segurança em que o suprimento sangüíneo para o fígado pode ser inter rompido em condições normais de temperatura, permanece desconhecido.
Em 1976, a ciclosporina, um poderoso agente imunossupressor, foi desenvolvida por Borel e introduzida na prática de transplante renal por CALNE et al. ${ }^{(2)}$. Estudos experimentais demonstraram que o tratamento com ciclosporina tinha efeito protetor na reperfusão de fígados submetidos a isquemia normotér mica ${ }^{(13)}$.

Atualmente, todos os métodos eficazes para preservação de órgãos para transplante baseiam-se na redução da temperatura como principal elemento protetor. Logo que o órgão fica isquêmico, seu suprimento de metabólitos cessa, podendo ocasionar lesões irreversíveis. Sabe-se que o simples

\footnotetext{
Departamento de Cirurgia, Faculdade de Medicina, Universidade Federal do Ceará; ${ }^{2}$ Hospital Universitário Walter Cantidio, Fortaleza, CE.

Endereço para correspondência: Dr. José Huygens P. Garcia - Programa de Pós-Graduação em Cir urgia - Rua Prof. Costa Mendes, 1608 - Rodolfo Teóf ilo

-60416-160 - Fortaleza, CE. E-mail: huygens@ secrel.com.br
} 
resfriamento tem sido bem-sucedido no sentido de reduzir esse dano, porém comporta muitas limitações. Portanto, caso as alterações metabólicas induzidas pela isquemia fossem mais detalhadamente estudadas, provavelmente seria possível intervir de forma ativa e conveniente nestes processos, prolongando a viabilidade hepática. Conseqüentemente, haveria melhora nos resultados do transplante hepático e de outros órgãos.

No presente estudo, foram determinadas as alterações nas concentrações hepáticas e sangüíneas de lactato, piruvato, glicose, corpos cetônicos, razão acetoacetato/3-hidroxibutirato e estado redox hepático citoplasmático e mitocondrial após 60 minutos de isquemia hepática normotérmica em ratos, assim como a provável ação hepatoprotetora da ciclosporina. Assim sendo, foram def inidos alguns aspectos do metabolismo hepático, contribuindo para a prevenção e tratamento da lesão isquêmica do fígado.

\section{MÉTODOS}

Foram utilizados 60 ratos albinos (Rattus norve gicus), variedade Wistar, machos, com peso médio de $300 \mathrm{~g}$, provenientes do biotério do Departamento de Fisiologia e Farmacologia da Uni versidade Federal do Ceará, Fortaleza, CE.

Os animais foram submetidos a anestesia inalatória com éter dietílico. Em seguida, foi realizada uma laparotomia mediana. O pedículo hepático, composto pela veia porta, artéria hepática e via biliar, foi isolado acima dos lobos caudado e lateral direito, sendo posteriormente ocluído com pinça vascular (Figura 1).A oclusão completa da tríade portal no local proposto é confirmada no transoperatório pela presença de palidez importante nos lobos mediano e lateral esquerdo, ao mesmo tempo em que os lobos inferiores não sujeitos à isquemia (caudado e lateral direito) preservam a coloração habitual. Após 60 minutos de pinçamento da tríade portal, que corresponde ao mesmo tempo de isquemia

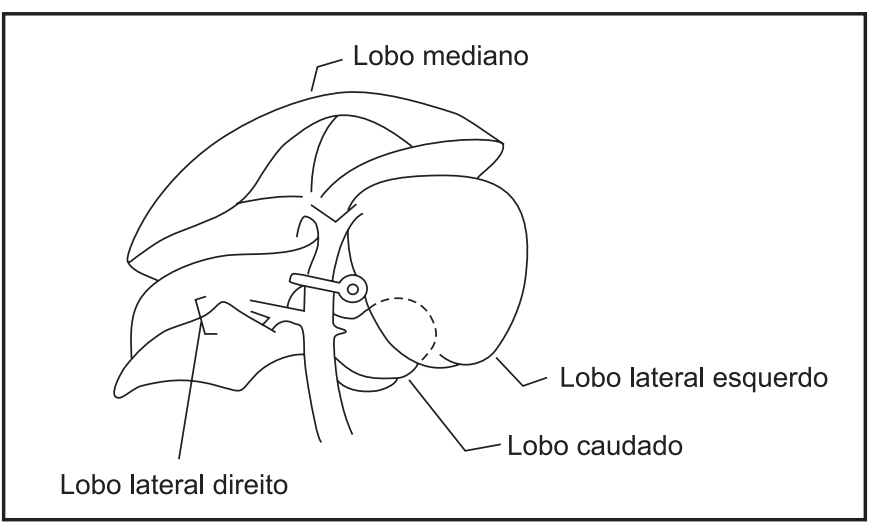

FIGURA 1 - Pinçamento seletivo do pedículo vascular hepática, os ratos foram novamente anestesiados e a ferida abdominal reaberta. Mais uma vez se confirma a eficácia da oclusão vascular pela isquemia dos lobos superiores. A pinça vascular foi retirada, observando-se a reperfusão dos lobos isquêmicos. Os lobos hepáticos não sujeitos à isquemia, foram, então, submetidos a ligadura de seus pedículos vasculares e ressecados (Figura 2). Desta forma, os animais permaneceram vivos somente com os lobos sujeitos à isquemia, permitindo estudar as alterações metabólicas induzidas pela isquemia, configurando o grupo isquêmico. Os animais do grupo-controle foram submetidos a ressecção dos lobos hepáticos inferiores sem oclusão vascular, de tal forma que permaneceram com a mesma quantidade de tecido hepático do grupo isquêmico.

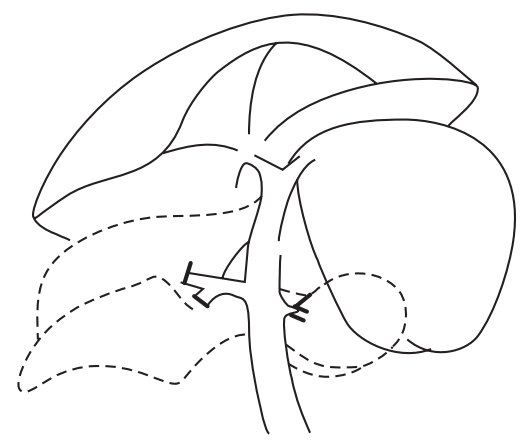

FIGURA 2 - Hepatectomia dos lobos não-isquemiados

Os animais foram divididos em 4 grupos de 12 unidades (6 controles e 6 isquêmicos). Todos os ratos do grupo isquêmico foram submetidos a 60 minutos de isquemia hepática normotérmica. Os animais do grupo-controle foram estudados nos mesmos tempos, porém sem isquemia hepática. $\mathrm{O}$ grupo 1 foi estudado no tempo 0 hora (isquêmico (I): imediatamente após 60 minutos de isquemia hepática; controle (C): após hepatectomia); o grupo 2 no tempo 1 hora (I: 1 hora após 60 minutos de isquemia hepática; $\mathrm{C}$ : 1 hora após hepatectomia); o grupo 3 no tempo 6 horas (I: 6 horas após 60 minutos de isquemia hepática; C: 6 horas após hepatectomia) e o grupo 4 no tempo 24 horas (I: 24 horas após 60 minutos de isquemia hepática; C: 24 horas após hepatectomia). Um quinto grupo foi tratado com administração intraperitone al de ciclosporina $(10 \mathrm{mg} / \mathrm{kg} / \mathrm{dia})$, por 4 dias antes da indução da isquemia, e estudado somente no tempo 1 hora (1 hora após 60 minutos de isquemia hepática nor motérmica). 

efeito hepatoprotetor da ciclosporina

Após serem anestesiados com éter e relaparotomizados, os ratos tiveram o sangue arterial colhido através de punção da aorta abdominal ( $1 \mathrm{~mL})$. Em seguida, o fígado foi removido, imediatamente prensado e mergulhado em nitrogênio líquido a cerca de 190 graus centíg rados negativos nos tempos $0,1,6$ e 24 horas. Tanto o tecido hepático, como o sangue arterial, foram submetidos a análise, por métodos enzimáticos, para determinação dos metabólitos: piruvato, acetoacetato, lactato, hidro xibutirato, glicose.

Os resultados foram expressos como média + EPM, acompanhada pelo número de observações (n). A significância estatística foi calculada de acordo com o teste $t$ de Student.

\section{RESULTADOS}

$\mathrm{Na}$ Tabela 1 são encontradas as alterações metabólicas das concentrações de lactato, piruvato, glicose, corpos cetônicos, razão acetoacetato/hidroxibutirato, estado redox mitocondrial e citoplasmático no fígado $(\mu \mathrm{mol} / \mathrm{g}$ ). No tempo 0 hora (g rupo 1$)$, houve aumento significante $(* P<0,05)$ das concentrações de lactato (C: 1,260 \pm $0,371 ;$ I: $7,584 \pm 1,264 *)$, glicose (C: $8,161 \pm 1,111$; I: $19,774 \pm$ $\left.1,837^{*}\right)$, e diminuição, também significante, da razão acetoacetato/ hidroxibutirato $(\mathrm{C}: 2,970 \pm 0,682$; I: $0,925 \pm 0,119 *)$, do potencial redox mitocondrial (C: 2053,60 $\pm 430,55$; I: $228,16 \pm 40,24 *)$ e citoplasmático (C: 60,24 \pm 13,92; I: 18,75 \pm 10,41*). Com 1 hora (grupo 2), houve elevação estatisticamente signif icante das concentrações de lactato (C: 3,920 \pm 0,359; I: $6,343 \pm 0,935^{*}$ ).
Na Tabela 2, em que foram avaliadas as alterações metabólicas das concentrações de lactato, piruvato, glicose, corpos cetônicos e razão acetoacetato/hidroxibutirato no sangue arterial $(\mu \mathrm{mol} / \mathrm{mL})$, ocorreram elevações significantes nas concentrações de glicose no tempo 0 hora (grupo 1) (C: 4,143 $\pm 0,676 ; \mathrm{I}: 7,039 \pm 0,713) \mathrm{e}$ 1 hora (grupo 2) (C: 5,738 $\pm 0,639$; I: 9,425 $\pm 1,439 *)$. Já com os corpos cetônicos (C: 0,160 $\pm 0,022$; I: $0,032 \pm 0,007 *)$ e razão acetoacetato/hidroxibutirato (C: 0,905 $\pm 0,193$; I: 0,035 $\pm 0,035^{* *}$, $P<0,0002 * *)$ houve diminuição importante 1 hora pós-isquemia (grupo 2).

$\mathrm{Na}$ Tabela 3, houve aumento significante, no tempo 1 hora (grupo 1), das concentrações dos corpos cetônicos (I: 0,032 \pm 0,007; I + ciclosporina (I + Cya): $0,142 \pm 0,020 * * *, P<0,005)$ e da razão acetoacetato/3-hidroxibutirato (I: $0,035 \pm 0,035$; I + Cya: $\left.0,193 \pm 0,036^{* * *}\right)$ no sangue arterial $(\mu \mathrm{mol} / \mathrm{mL})$ em resposta ao tratamento com ciclosporina quando comparado com o grupo submetido a isquemia.

\section{DISCUSSÃO}

Os resultados do presente estudo vão ao encontro dos resultados da literatura, pois evidenciaram aumento do lactato hepático na fase aguda após revascularização, particularmente nos primeiros minutos ${ }^{(6)}$. No tempo 0 hora (imediatamente após 60 minutos de isquemia hepática sem revascularização), cerca de $30 \%$ do fígado (lobo lateral direito e caudado) permaneceu perfundido e, portanto, funcionante.

TABELA 1 - Alterações com o tempo nas concentrações de lactato, piruvato, glicose, corpos cetônicos, razão acetoacetato/hidroxibutirato, estado redox mitocondrial e citoplasmático no fígado $(\mu \mathrm{mol} / \mathrm{g}) \mathrm{n}=6$ para cada grupo

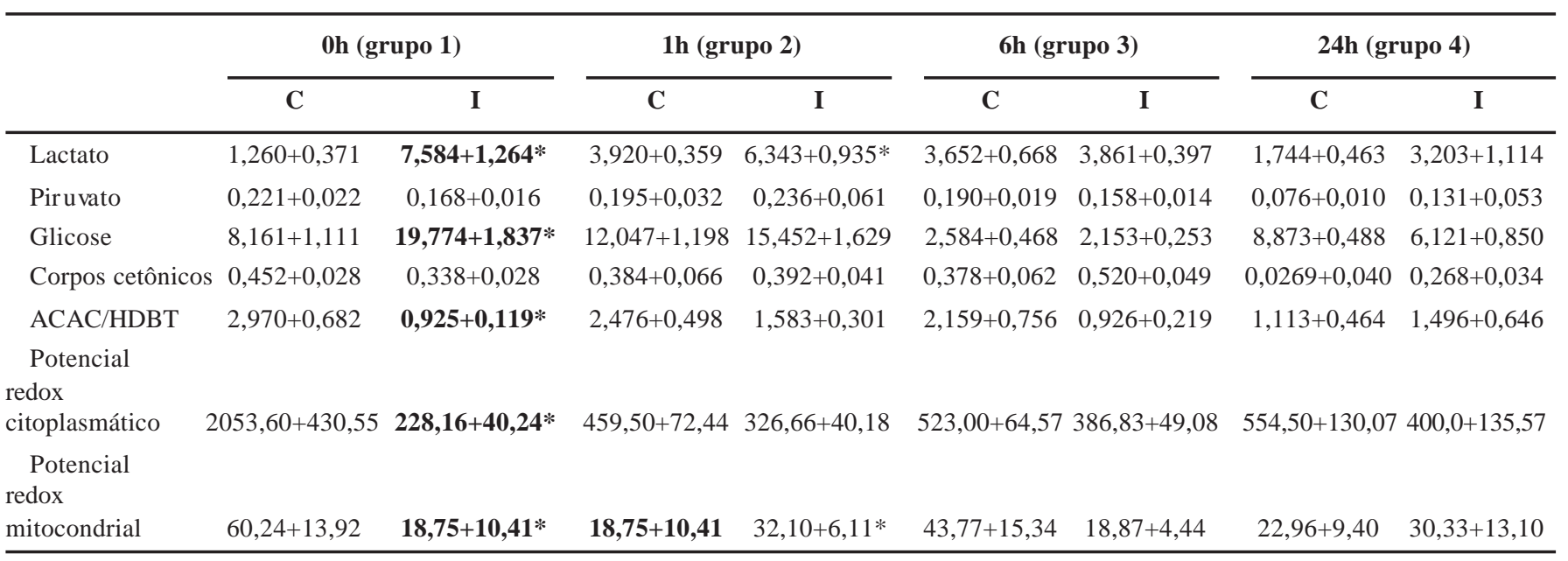

Os resultados são e xpressos como média + EPM

$\mathrm{C}=$ controle; $\mathrm{I}=$ isquemia

$\mathrm{ACAC} / \mathrm{HDBT}=$ razão acetoacetato/hidroxibutirato

$* P<0,05$ em relação ao controle 
Garcia JHP, Coelho GR, Sousa IT, Siqueira RP, Vasconcelos PRL. Alterações metabólicas induzidas por isquemia hepática normotérmica experimental e o efeito hepatoprotetor da ciclosporina

TABELA 2 - Alterações com o tempo nas concentrações de lactato, piruvato, glicose e corpos cetônicos e razão acetoacetato/hidroxibutirato no sangue arterial $(\mu \mathrm{mol} / \mathrm{mL}) \mathrm{n}=6$ para cada grupo

\begin{tabular}{|c|c|c|c|c|c|c|c|c|}
\hline & \multicolumn{2}{|c|}{ Oh (grupo 1) } & \multicolumn{2}{|c|}{ 1h (grupo 2) } & \multicolumn{2}{|c|}{ 6h (grupo 3) } & \multicolumn{2}{|c|}{ 24h (grupo 4) } \\
\hline & $\mathrm{C}$ & I & $\mathbf{C}$ & I & $\mathrm{C}$ & I & $\mathrm{C}$ & I \\
\hline Lactato & $1,085+0,423$ & $1,845+0,380$ & $2,460+0,399$ & $4,461+0,937$ & $2,298+0,597$ & $2,463+0,359$ & $1,827+0,277$ & $2,529+0,580$ \\
\hline Piruvato & $0,172+0,013$ & $0,104+0,036$ & $0,058+0,025$ & $0,026+0,006$ & $0,112+0,026$ & $0,088+0,015$ & $0,084+0,009$ & $0,137+0,036$ \\
\hline Glicose & $4,143+0,676$ & $7,039+0,713 *$ & $5,738+0,639$ & $9,425+1,439 *$ & $1,680+0,299$ & $1,477+0,122$ & $2,076+0,326$ & $2,076+0,714$ \\
\hline Corpos cetônicos & $0,133+0,016$ & $0,089+0,024$ & $0,160+0,022$ & $0,032+0,007 *$ & $0,170+0,025$ & $0,157+0,026$ & $0,198+0,048$ & $0,244+0,046$ \\
\hline ACAC/HDBT & $2,406+1,102$ & $1,488+0,857$ & $0,905+0,193$ & $0,035+0,035 * *$ & $0,436+0,085$ & $0,294+0,050$ & $0,365+0,137$ & $0,267+0,124$ \\
\hline
\end{tabular}

Os resultados são expressos como média + EPM

$\mathrm{C}=$ controle; $\mathrm{I}=$ isquemia

$\mathrm{ACAC} / \mathrm{HDBT}=$ razão acetoacetato/hidroxibutirato

$* P<0,05$ em relação ao controle

**P $P<0,0002$ em relação ao controle

TABELA 3 - Alterações nas concentrações dos corpos cetônicos no sangue arterial $(\mu \mathrm{mol} / \mathrm{mL})$ e na razão acetoacetato/3-hidroxibutirato em resposta ao tratamento com ciclosporina após $1 \mathrm{~h}$ de reperfusão hepática $\mathrm{n}=6$ para cada gr upo

\begin{tabular}{lcccc}
\hline & C & I & C + Cya & I + Cya \\
\hline Corpos cetônicos & $0,160+0,022$ & $0,032+0,007$ & $0,119+0,018$ & $\mathbf{0 , 1 4 2 + 0 , 0 2 0 *}$ \\
ACAC/HDBT & $0,095+0,193$ & $0,035+0,035$ & $0,678+0,172$ & $\mathbf{0 , 1 9 3 + 0 , 0 3 6 *}$ \\
\hline
\end{tabular}

Os resultados são expressos como média + EPM

$\mathrm{C}=$ controle; $\mathrm{I}=$ isquemia; $\mathrm{Cya}=$ ciclosporina

ACAC/HDBT = razão acetoacetato/hidroxibutirato

$* P<0,005$ em relação à isquemia sem tratamento

Desta forma, houve metabolização do lactato, e vitando o seu acúmulo na circulação sangüínea. Isto pode explicar a ausência de hiperlactacemia neste tempo estudado (Tabelas 1,2).

Os níveis hepáticos de lactato caíram com o tempo, podendo indicar recuperação progressiva dos hepatócitos quando o fluxo sangüíneo é restaurado. É possível que o acúmulo progressivo de lactato no fígado, durante a isquemia hepática, possa ser um dos fatores responsáveis por dano celular irreversível, visto que nos experimentos de FARKOUH et al. ${ }^{(5)}$ nenhum cão sobre viveu quando os níveis deste metabólito ultrapassaram $17 \mu \mathrm{mol} / \mathrm{g}$ de tecido hepático (Tabela 2).

A isquemia hepática elevou consideravelmente a concentração de glicose no fígado somente no tempo 0 hora. No fígado isquêmico pode haver ativação da fosforilase hepática com incremento da glicogenólise, tal como acontece no músculo cardíaco ${ }^{(11)}$. Portanto, a glicogenólise ativada pode explicar a elevação da concentração de glicose hepática (Tabela 1).

Nos tempos 1 hora, 6 horas e 24 horas pós-isquemia não houve diferença nas concentrações de glicose hepática entre os grupos controle e isquêmico, provavelmente pela reoxigenação do tecido hepático com retorno da respiração celular e conseqüente diminuição da glicogenólise.

As glicemias sangüíneas se elevaram nos tempos 0 hora e 1 hora. Tal fato se deve, provavelmente, ao bloqueio de captação de glicose pelo fígado isquêmico e pela liberação para circulação da glicose armazenada a partir do glicogênio hepático (Tabela 2).

A isquemia hepática acarretou queda acentuada da concentração dos corpos cetônicos e da razão acetoacetato/3-hidroxibutirato no sangue no tempo 1 hora pós-isquemia, possivelmente decorrente da redução da síntese destes metabólitos. Com o decorrer do tempo (6 horas e 24 horas), esse valores voltaram a níveis semelhantes aos encontrados no grupo-controle, sugerindo recuperação do hepatócito após injúria isquêmica. No fígado houve diminuição significativa da razão dos corpos cetônicos no tempo 0 hora. É provável que neste tempo de estudo, o tecido hepático, que permaneceu vascularizado (lobo lateral e caudado), tenha sido responsável pelo balanço e conseqüente normalização da razão dos corpos cetônicos na circulação arterial (Tabelas 1,2).

A isquemia hepática causou queda tanto no estado redox citoplasmático, como do mitocondrial no tempo 0 hora. Desta forma, 

efeito hepatoprotetor da ciclosporina

houve redução entre estes dois compartimentos na razão [NAD+]/ [NADH], dificultando o transpor te de elétrons e, conseqüentemente, a formação de $\operatorname{ATP}^{(12)}$. Nos tempos seguintes (1 hora, 6 horas, 24 horas), a revascularização parece ter restaurado o estado redox das células hepáticas, visto que não houve diferenças entre animais isquêmicos e os grupos de controle (Tabela 1).

A ciclosporina causou elevação da razão dos corpos cetônicos no sangue arterial, mas não se elevou no tecido hepático. Portanto, o aumento da razão acetoacetato/3-hidroxibutirato no sangue não refletiu a recuperação da função mitocondrial hepática. Deste modo, o efeito protetor da ciclosporina na injúria pós-isquêmica do fígado poderia estar relacionada ao aumento da capacidade de sintetizar corpos cetônicos, já que houve elevação das concentrações destes metabólitos no sangue no tempo estudado. Este efeito protetor da ciclosporina pode estar relacionado à inibição da aber tura dos canais de cálcio dependentes da membrana interna mitocondrial associados à produção diminuída de radicais livres de oxigênio ${ }^{(3,8)}$. O efeito protetor da ciclosporina na prevenção de lesões hepáticas ocasionadas por isquemia é também devido à modulação da produção de fator de necrose tumoral $(\mathrm{FTN})^{(9,10)}$. O aumento dos níveis de FTN pré-transplante está relacionado com rejeição aguda póstransplante ${ }^{(1)}$. Assim sendo, é pertinente especular que a utilização de ciclosporina em receptores antes do transplante ou seu uso em soluções de preservação, poderá melhorar os resultados do transplante hepático.

\section{CONCLUSÕES}

A isquemia hepática ocasionou aumento significativo das concentrações de lactato no fígado, sugerindo pronunciado grau de metabolismo anaeróbico durante o período de isquemia e o período inicial de reperfusão.

Houve elevação da concentração de glicose no fígado, provavelmente por ativação da enzima fosforilase e conseqüente glicogenólise. No sangue houve hiperglicemia, provavelmente resultante do bloqueio de captação de glicose pelo fígado isquêmico e pela liberação para circulação da glicose ar mazenada a partir do glicogênio hepático.

A concentração dos corpos cetônicos e a razão acetoacetato/ 3-hidroxibutirato no sangue caíram, possi velmente devido à redução de síntese destes metabólitos.

O estado redox citoplasmático e mitocondrial das células hepáticas diminuiu somente no tempo 0 hora, sugerindo que a revascularização com normalização da oferta de oxigênio recupera rapidamente o estado redox.

A ciclosporina causou elevação significante das concentrações dos corpos cetônicos e da razão acetoacetato/3-hidroxibutirato no sangue arterial, podendo refletir recuperação da função hepática.

Garcia JHP, Coelho GR, Sousa IT, Siqueira RP, Vasconcelos PRL. Induced metabolic alterations due to experimental normothermic hepatic ischemia and the hepatoprotector effect of cyclosporin. Arq Gastroenterol 2004;41(1):54-59.

ABSTRACT - Background - Hepatic transplantation is inevitably associated with periods of complete ischemia. However, the clamping of hepatic vascular pedicle is limited by the consequences of the post-ischemic injury to the liver. Aim - To deter mine the main metabolic alterations caused for the hepatic ischemia and the probable hepatoprotecti ve effect cyclosporin. Method - Normothermic hepatic ischemia during 60 minutes was induced in the rats. The time-course $(0,1,6,24$ hours) of changes in blood and in the hepatic concentrations of lactate, pyruvate, glucose, ketone bodies and in the ratio of acetoacetate/3-hydroxybutyrate, as well as the cytoplasmic and mitochondrial redox state of the liver cells were determined. A group of animals was daily pre-treated with cyclosporine $(10 \mathrm{mg} / \mathrm{kg}$ ) during 4 days until the induction of hepatic ischemia, then the y studied 1 hour after hepatic revascularization. Hepatic ischemia caused elevation in the concentrations of lactate in the liver, suggesting that a pronounced level of anaerobic metabolism occurred during the ischemia period. Liver ischemia promoted yet a fall in the concentration and in the ratio of ketone bodies (acetoacetate/3-hydroxybutyrate) in the ar terial blood in the studied period of one hour post-revascularization, perhaps reflecting impairment of ketogenesis as a result of the ischemic injury. Conclusion - The treatment with cyclosporine cause ele vation in the concentration of ketone bodies and in the ratio of acetoacetate/3-hydroxybutyrate in the arterial blood 1 hour after reperfusion of the liver, suggesting that these drugs may accelerate the recovery of the ischemic hepatic lesion with reactivation of ketogenesis.

HEADINGS - Liver. Hepatectomy. Ischemia. Cyclosporin. Rats. 


\section{REFERÊNCIAS BIBLIOGRÁFICAS}

1. Bathg ate AJ, Lee P, Hayes P, Simpson KJ. Pretransplantation tumor necrosis factor-alpha production predicts acute rejection after liver transplantation. Liver Transpl 2000;6:721-7.

2. Calne RY. Transplantation of the li ver. Ann Surg 1978;188:129.

3. Crompton M, Ellinger H, Costi A. Inhbition by cyclosporin A of a $\mathrm{Ca} 2+$ dependent pore in hear $\mathrm{t}$ mitochondria activated by inorg anic and oxidati ve stress. Biochem J 1988;255:357.

4. Delva E, Camus Y, Nordlinger B, Hannoun L, Parc R, Deriaz H, Lienhart A, Uguet C. Vascular occlusions for liver resections: operative management and tolerance to hepatic ischemia. Ann Surg 1989;209:211-8.

5. Farkouh E, Daniel AM, Beaudoin JG, McLean LD. Predictive value of live biochemistry in acute hepatic ischemia. Surg Gynecol Obstet 1971;5:832-8.

6. Harris KA, Wallace AC, Wall WJ . Tolerance of the liver to ischemia in the pig J Surg Res 1982;33:524-30.

7. Huguet $\mathrm{C}$, Nordlinger B, Bloch P, Conard J. Tolerance of the human liver to prolonged normother mic ischemia. Arch Surg 1978;113:1448-51.
8. Igbavboa U, Zwizinski CW, Pfeiffer DR. Release of mitochondrial matrix proteins through a $\mathrm{Ca} 2+$ requiring, cyclosporin-sensitive pathway. Biochem Biophys Res Commun 1989;161:619.

9. Ishii T, Kim YI, Kawano K, Tatsuma T, Shimada T, Kitano S. Amelioration of tumor necrosis factor release by cyclosporine in wam ischemia/reperfusion injury of the rat li ver: with special reference to hepatic ultrastructure. J Hepatobiliary Pancreat Surg 1999;6:267-74.

10. Mizuta K, Ohmori M, Miyashita F, Kitoh Y, Fujimura A, Mori M, Kanno T, Hashizume. Effect of pretreatment with FTY720 and cyclosporin on ischaemiareperfusion injury of the liver in rats. J Pharm Pharmacol 1999;51:1423-8.

11. Newsholme EA, Start C. The $[\mathrm{NAD}+] /[\mathrm{NADH}]$ ratios in the cytoplasmic and mitochondrial compartments of the liver cell. In: Newsholme EA, Start C, editores. Regulation in metabolism. ENGLAND JW. Ar rowsmith Bristol 1981;7:324-8.

12. Ne wsholme EA, Start C. Re gulation of glycogen metabolism. In: Newsholme EA, Start C. Re gulation in metabolism. London: Wiley; 1981. p.300-23.

13. Travis DL, Fabia R, Netto GG, Husberg BS, Goldstein RM, Klintmalm GB Levy MF. Protection by cyclosporine A ag ainst normothermic liver ischemiareperfusion in pigs. J Surg Res 1998;75:116-26.

Recebido em 23/12/2002 Aprovado em 10/7/2003. 